\title{
CCN3 (NOV) regulates proliferation, adhesion, migration and invasion in clear cell renal cell carcinoma
}

\author{
SHUAI LIU* ${ }^{*}$ ZHENG LIU*, DONGBIN BI, XAODONG YUAN, XIAOWEN LIU, \\ SENTAI DING, JIAJU LU and ZHIHONG NIU \\ Department of Urology, Provincial Hospital Affiliated to Shandong University, Jinan 250021, P.R. China
}

Received November 14, 2011; Accepted February 8, 2012

DOI: 10.3892/ol.2012.607

\begin{abstract}
The CCN3/nephroblastoma overexpressed gene belongs to the $\mathrm{CCN}$ family of genes that encode secreted proteins involved in a variety of processes including tumorigenesis. Altered expression of CCN3 has been observed in human nephroblastoma and renal cell carcinoma (RCC), suggesting that $\mathrm{CCN} 3$ plays a role in kidney tumorigenesis. The aim of the present study was to examine the role of CCN3 in clear cell RCC biology. In particular, we studied the expression of CCN3 in 32 pairs of RCC tissues and corresponding normal kidney tissues using immunohistochemistry. The CCN3 gene was transfected into the 786-O cell line and the behaviors of stably transfected clones were analyzed. Results showed the expression of CCN3 was lower in RCC tissues compared to corresponding normal kidney tissues and the expression of CCN3 was inversely correlated with the Ki67 index. CCN3-expressing clones exhibited significantly inhibited cell proliferation. Furthermore, CCN3-transfected 786-O cells exhibited increased adhesion to extracellular matrix proteins, migration and invasion in Matrigel. Our data indicated that CCN3 plays an anti-proliferative role in clear cell RCC cells and promotes the adhesion, migration and invasion of clear cell RCC cells.
\end{abstract}

\section{Introduction}

Renal cell carcinoma (RCC) is the most common type of cancer of the kidney. Metastasis frequently becomes evident at the time of diagnosis or patients develop distant metastases following removal of the primary tumor. The treatment of metastatic, locally unresectable RCCs remains a challenge for urologists (1). There is a great need to understand the basic biology of RCC and develop better therapeutic options.

Correspondence to: Professor Zhihong Niu, Department of Urology, Provincial Hospital affiliated to Shandong University, 324 Jingwu Street, Jinan 250021, P.R. China

E-mail:nzh1789@yahoo.com

*Contributed equally

Key words: renal cell carcinoma, $\mathrm{CCN} 3$, migration, tumorigenicity
The CCN3 (nephroblastoma overexpressed, NOV) gene belongs to the $\mathrm{CCN}$ family of genes, which has five additional members: cystein-rich protein 61 (Cyr61), connective tissue growth factor (CTGF), Wnt-1-induced secreted protein (WISP)-1, WISP-2 and WISP-3. CCN proteins are involved in fundamental biological processes such as cell proliferation, attachment, migration, differentiation, wound healing, angiogenesis and tumorigenesis (2).

The CCN3 protein is detectable at varying levels in the kidney, muscle, cartilage, brain, lung, ovary and heart (3-5). The functions of $\mathrm{CCN} 3$ protein among these different tissues are, however, different. CCN3 was originally described as antiproliferative (3), and its expression was associated with differentiation of Wilms' tumor (4), rhabdomyosarcomas (6), neuroblastomas (7), cartilaginous tumors (8), adrenocortical tumors (9) and with inhibition of the growth and decrease in tumorigenicity of several tumor cell lines including glioblastoma (10), choriocarcinoma (11) and Ewing's sarcoma (12). Furthermore, CCN3 expression was correlated with the increased proliferative index of $3 \mathrm{~T} 3$ fibroblast (13) and tissue samples of the prostate (14). Although $\mathrm{CCN} 3$ reduced the growth rate of Ewing's sarcoma transfectants ex vivo, CCN3 expression was associated with poor prognosis and shown to increase cell motility, resulting in enhanced metastatic potential $(6,12)$.

In the present study, we confirm that the expression of CCN3 protein is decreased in clear cell RCC tissues compared to normal kidney tissues. To further investigate the role of CCN3 in the biology of clear cell RCC cells, we transfected 786-O cells with the CCN3 gene. The effects of CCN3 on cell proliferation, adhesion, migration and invasion are described.

\section{Materials and methods}

Human tissues. RCC tissues were obtained from 32 patients with clear cell RCC (median age, 65 years; range, $45-83$ years) treated by radical nephrectomy at Shandong Provincial Hospital (Jinan, China). Samples were fixed in $4 \%$ buffered formalin, embedded in paraffin, and routinely stained with hematoxylin and eosin (H\&E). Corresponding normal kidney tissues were also obtained from these patients. Our study was approved by the Ethics Committee of the Shandong Provincial Hospital.

Immunohistochemistry. Formalin-fixed, paraffin-embedded $\mathrm{RCC}$ and corresponding normal kidney tissue sections $(5 \mu \mathrm{m})$ 
were deparaffinized with xylene, rehydrated and washed with phosphate-buffered saline (PBS). Endogenous peroxidase activity was blocked with $3 \% \mathrm{H}_{2} \mathrm{O}_{2}$ for $20 \mathrm{~min}$. Antigen retrieval was performed in a $0.01 \mathrm{M}$ sodium citrate buffer in a microwave oven. Slides were blocked with $10 \%$ bovine serum for $1 \mathrm{~h}$, followed by incubation with primary antibodies at $4^{\circ} \mathrm{C}$ overnight. The primary antibodies used were: rabbit polyclonal to CCN3 (Abcam, Cambridge, UK, 1/2000) and rabbit polyclonal to Ki67 (Abcam, 1/50). After washing in $0.01 \mathrm{M} \mathrm{PBS}$, the slides were incubated with biotinylated goat anti-rabbit IgG (1:50) for $30 \mathrm{~min}$, then stained with $0.03 \%$ diaminobenzidine for $10 \mathrm{~min}$. Slides were counterstained with hematoxylin. The stain was evaluated by two independent assessors. The staining intensity for CCN3 was expressed as follows: -, no stain; +, weak; ++, moderate; and +++, strong. After staining with Ki67 antibody, cells were enumerated by counting the number of positive cells in 10 fields over the total cells at x400 magnification.

Cell lines and transfections. The RCC cell line (786-O cell line) was purchased from the cell bank of the Chinese Academy of Sciences. We constructed a plasmid based on the PCMV-NOVH plasmid which contains human CCN3 under the control of a constitutive or inducible promoter (kindly provided by Dr Cecile Martinerie, Hospital Saint-Antoine, France). To monitor the transfection rate by fluorescence microscopy, EGFP and Neo, which encode enhanced green fluorescence protein and Neomycin resistance protein, respectively, were inserted to make the construct (pEGFP-C1-CCN3), and the plasmid was verified by sequencing. The 786-O cell line was transfected with this vector and Lipofectamine 2000 (Invitrogen, Carlsbad, CA, USA). A fluorescence microscope was used to monitor the transfection rate. Stable transfectants were selected in G418 (800 $\mu \mathrm{g} / \mathrm{ml}$, Invitrogen) and characterized for CCN3 expression level by real-time reverse transcriptase-polymerase chain reaction (RT-PCR) analysis and western blotting, respectively. Among the clones obtained, one line with significantly higher levels of CCN3 expression was selected and compared with the parental 786-O cell line. Cells transfected with the empty vector $\mathrm{pEGFP-C1}$ were used as negative controls.

Real-time RT-PCR analysis. Quantitative real-time RT-PCR was carried out using SYBR-Green according to the standard protocol (Roche Diagnostics GmbH, Mannheim, Germany). Briefly, total RNA was isolated, then cDNA was reverse transcribed from $1 \mu \mathrm{g}$ of RNA by RT-PCR standard methods. Real-time PCR reactions were carried out in triplicate on an ABI 7500 machine using gene expression assays (Applied Biosystems, Foster City, CA, USA). The real-time PCR primers for CCN3 were 5'-GCATCTGCACGGCGGTAG-3' (forward) and 5'-CACTGGAATTTGCAGCTTGG-3' (reverse). PCR products were detected by measuring the emitted fluorescence at the end of each reaction step (reaction cycle). The threshold cycle $(\mathrm{Ct})$ corresponds with the cycle number required to detect a fluorescence signal above the baseline. We used the housekeeping gene glyceraldehyde-3-phosphate dehydrogenase (GAPDH) as an endogenous control and the 786-O cell line as a calibrator. A negative control without a cDNA template was run with each assay. Data analysis was carried out using SDS version 1.3.0.
Western blot analysis. To determine the expression of CCN3, proteins were extracted from cells via suspension in RIPA buffer. Samples were centrifuged at $10,000 \mathrm{x}$ g at $4^{\circ} \mathrm{C}$ for $10 \mathrm{~min}$ and the supernatant was used for analysis. The protein concentration was determined using the Bradford protein method and the BCA protein assay kit (Sigma, St Louis, MO, USA). Protein $(50 \mu \mathrm{g})$ was loaded onto a pre-cast Bis-Tris polyacrylamide gel (8\%) and subsequently transferred to a PVDF membrane, then blotted with anti-CCN3 rabbit polyclonal antibodies, rabbit anti-actin (Abcam) and (HRP)-conjugated secondary antibodies (Abcam). Immunoblots were visualized by enhanced chemiluminescence (LAS4000).

Growth curve. Cells were trypsinized, and $1.5 \times 10^{4}$ cells were plated in each well of 24-well plates containing RPMI-1640 with FBS at a concentration of $10 \%$. Every $24 \mathrm{~h}$, the medium was removed, adherent cells were trypsinized, and total adherent cells in each well were quantified using a hematocytometer. Cell counts of 3 wells per time point per group were averaged and the data were used to draw growth curves.

WST-1 assay for cell proliferation. For WST-1 proliferation assays (WST-1 cell proliferation and cytotoxicity assay kit; Beyotime, Institute of Biotechnology, Haimen, China), cells in an exponential phase of growth were harvested and seeded in 96-well plates at a density of 2,000 cells per well, and cultured for 24,48 , and $72 \mathrm{~h}$ at $37^{\circ} \mathrm{C}$ with $5 \% \mathrm{CO}_{2}$. Following the culture, $10 \mu 1$ WST-1 kit was added into each well. Plates were then incubated for an additional $1 \mathrm{~h}$ period at $37^{\circ} \mathrm{C}$. Subsequently, absorbance was determined in a microplate reader (EL340 Bio-TEK instruments, Hopkinton, MA, USA) at $450 \mathrm{~nm}$.

Cell adhesion assays. The effect of CCN3 expression on the adhesive properties of RCC cells was analyzed using different ECM (extracellular matrix) substrates, such as laminin (LN) and fibronectin (FN). Cell adhesion to laminin and fibronectin was tested on tissue culture plates, which were coated overnight at $4^{\circ} \mathrm{C}$ with $0.2 \mu \mathrm{g} / \mathrm{cm}^{2}$ human placental laminin and fibronectin (Sigma), then blocked with $1 \%$ bovine serum albumin at room temperature for $1 \mathrm{~h}$. Cells $\left(10^{5} / \mathrm{ml}\right.$ x $\left.200 \mu \mathrm{l}\right)$ were plated and incubated at $37^{\circ} \mathrm{C}$ for $90 \mathrm{~min}$, non-adherent cells were rinsed off with RPMI, and adherent cells were fixed with methanol for $15 \mathrm{~min}$ and stained with $0.1 \%$ crystal violet. Plates were washed by immersion in tap water and then dried, and absorbance at $550 \mathrm{~nm}$ was measured following cell solubilization with a 50:50 mixture of $0.1 \mathrm{~mol} / 1 \mathrm{NaH}_{2} \mathrm{PO}(\mathrm{pH} \mathrm{4.5)}$ in $50 \%$ ethanol. The experiment was performed in triplicate.

Transwell migration assay. Transwell chambers (Costar, Cambridge, UK) with $8-\mu \mathrm{m}$ pore size polycarbonate filter inserts for 24-well plates were used according to the manual. In brief, $1 \times 10^{5}$ cells in serum-free medium (RPMI-1640) were plated in the upper well of the transwell chambers, whereas medium with $10 \%$ fetal bovine serum was added to the lower well. Following a 12-h incubation, the cells on the upper side of the inserts were removed using a cotton swab. The inserts were fixed in methanol and stained with $\mathrm{H} \& \mathrm{E}$. The number of migrated cells attached to the other side of the insert was counted under a light microscope in 8 random fields at a magnification of $\mathrm{x} 200$. The experiment was performed in triplicate. 

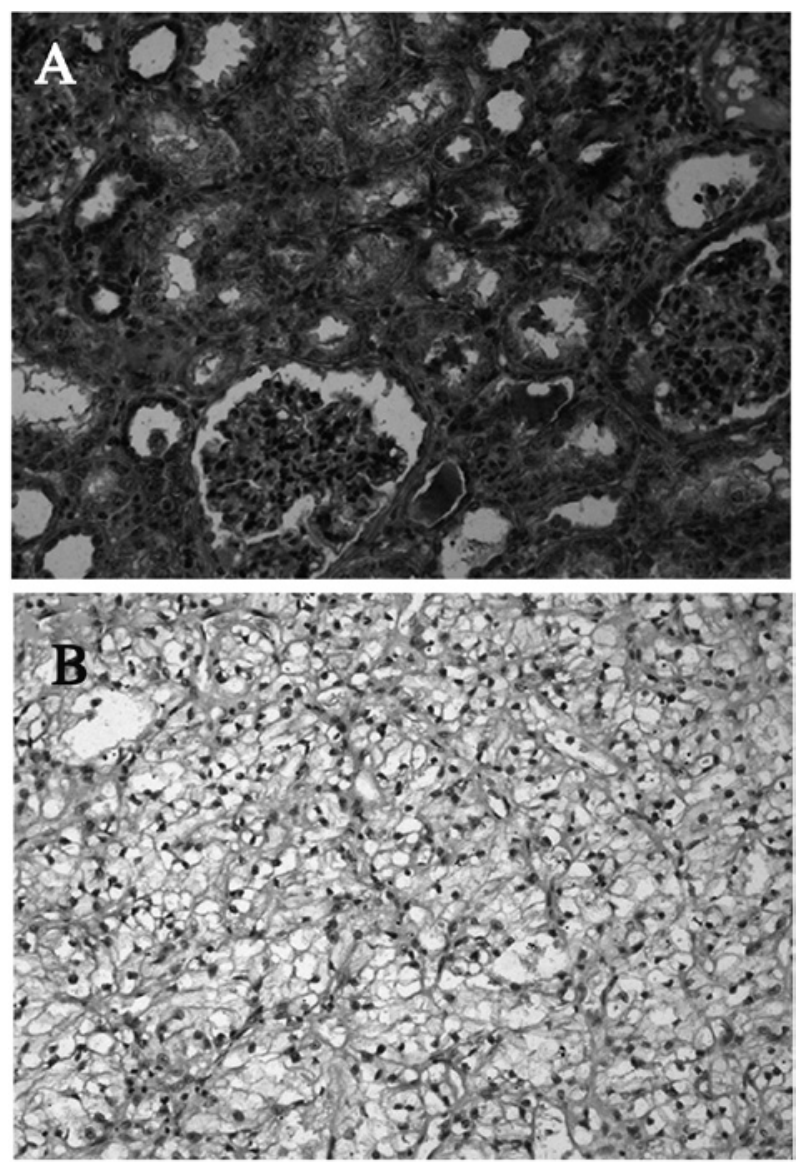

Figure 1. Representative immunohistochemical staining of $\mathrm{CCN} 3$ in (A) normal kidney tissue and (B) clear cell RCC is shown. Original magnification, $\mathrm{x} 200$.

Matrigel invasion assay. Invasion assays were performed using Matrigel (BD Bioscience, Bedford, MA, USA)-coated transwell chambers. Briefly, the transwell inserts were precoated with $40 \mu \mathrm{l}$ of $1 \mathrm{mg} / \mathrm{ml}$ Matrigel matrix. Cells $\left(1 \times 10^{5}\right)$ in serum-free medium were plated in the upper well, whereas medium with $10 \%$ fetal bovine serum was added to the lower well. After incubating for $24 \mathrm{~h}$, the cells on the Matrigel side of the chambers were removed with a cotton swab. The inserts were fixed in methanol and stained by H\&E staining. The number of invaded cells attached to the other side of the inserts was counted under a light microscope in 8 random fields at a magnification of $\mathrm{x} 200$. The experiment was performed in triplicate.

Statistical analysis. Results are presented as the means \pm SD. Statistical analyses included the Student's t-test, performed using the Statistical Package for Social Science (SPSS for Windows package release 10.0; SPSS Inc., Chicago, IL, USA) as indicated in Results and figure legends. $\mathrm{P}<0.05$ was considered to represent a statistically significant result.

\section{Results}

Immunohistochemistry. In all cases, the adjacent normal kidney tissue demonstrated intense CCN3 immunostaining. CCN3 was localized in the cytoplasm of the renal tubules
Table I. Immunostaining intensity of CCN3 in RCC tissues.

\begin{tabular}{|c|c|c|c|c|}
\hline No. & Grade $^{a}$ & T stage $^{\mathrm{b}}$ & $\mathrm{CCN} 3$ & $\operatorname{Ki67}(\%)$ \\
\hline 1 & 2 & 1 & + & 2.4 \\
\hline 2 & 2 & 2 & - & 0.5 \\
\hline 3 & 3 & 2 & - & 5.6 \\
\hline 4 & 1 & 2 & + & 1.5 \\
\hline 5 & 1 & 1 & - & 2.2 \\
\hline 6 & 2 & 2 & + & 4.2 \\
\hline 7 & 2 & 1 & + & 2.8 \\
\hline 8 & 2 & 1 & ++ & 0.6 \\
\hline 9 & 3 & 2 & - & 3.3 \\
\hline 10 & 2 & 2 & + & 2.7 \\
\hline 11 & 1 & 1 & ++ & 0.2 \\
\hline 12 & 3 & 2 & + & 2.8 \\
\hline 13 & 2 & 1 & - & 2.4 \\
\hline 14 & 2 & 1 & + & 1.7 \\
\hline 15 & 2 & 1 & - & 2.8 \\
\hline 16 & 1 & 2 & + & 0.5 \\
\hline 17 & 2 & 2 & ++ & 2.8 \\
\hline 18 & 1 & 2 & + & 0.3 \\
\hline 19 & 2 & 3 & + & 0.5 \\
\hline 20 & 2 & 2 & ++ & 2.9 \\
\hline 21 & 2 & 3 & +++ & 3.1 \\
\hline 22 & 2 & 2 & - & 1.7 \\
\hline 23 & 3 & 3 & ++ & 2.4 \\
\hline 24 & 1 & 3 & + & 0.5 \\
\hline 25 & 2 & 3 & ++ & 0.4 \\
\hline 26 & 2 & 3 & - & 5.9 \\
\hline 27 & 3 & 3 & - & 4.5 \\
\hline 28 & 3 & 3 & ++ & 0.8 \\
\hline 29 & 2 & 2 & ++ & 1.6 \\
\hline 30 & 3 & 3 & +++ & 1.2 \\
\hline 31 & 2 & 2 & - & 1.5 \\
\hline 32 & 2 & 2 & - & 3.2 \\
\hline
\end{tabular}

${ }^{\mathrm{a}}$ Fuhrman classification; ${ }^{\mathrm{b}} \mathrm{AJCC} 2002$ TNM classification.

and parietal epithelial cells of the glomeruli (Fig. 1A). CCN3 staining was observed in 21/32 (65.6\%) of clear cell RCCs. The intensity of CCN3 staining was generally less pronounced than in the corresponding normal kidney tissue. The majority of cell membrane staining was observed in tumor cell RCCs (Fig. 1B). The proportion of Ki67-positive tumor cells was significantly higher in the $\mathrm{CCN} 3$-negative tumors $(3.1 \pm 1.7 \%)$ compared to the CCN3-positive tumors $(1.7 \pm 1.2 \%)(\mathrm{P}<0.05)$ (Table I). However, no obvious correlations were observed between the intensity of staining for $\mathrm{CCN} 3$ and tumor stage or grade.

CCN3 expression changed by plasmid transfection. To determine the CCN3 expression level in 786-O cells with CCN3 plasmid transfection, quantitative real-time RT-PCR analysis and western blot analysis of $\mathrm{CCN} 3$ were measured in CCN3-transfected 786-O cells, mock transfectants and parental 786-O cells. The expression of CCN3 mRNA and protein was 
A

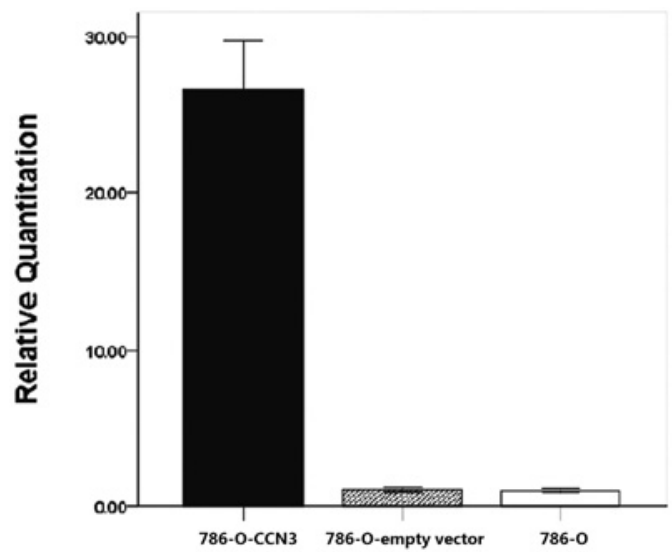

B

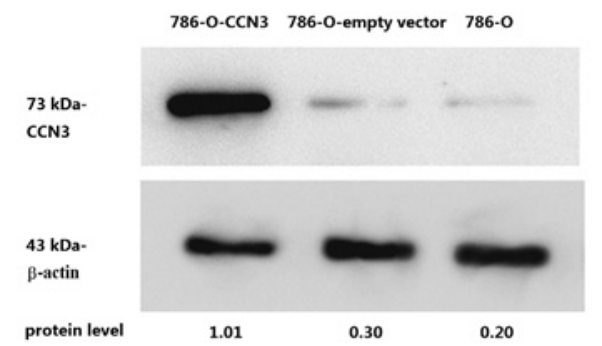

Figure 2. Expression of CCN3 in CCN3-transfected 786-O cells. (A) Relative expression of CCN3 mRNA in CCN3-transfected clones with respect to the parental $786-\mathrm{O}$ cell line used as a calibrator $\left(2^{-\Delta \Delta C t}=1\right)$, by real-time PCR . Triplicate experiments for each sample were performed and results are shown as the mean $\pm \mathrm{SD}$. ${ }^{*} \mathrm{P}<0.05$. (B) Increased expression of $\mathrm{CCN} 3$ in $\mathrm{CCN} 3$ transfected clones was confirmed by western blot analysis using a polyclonal antibody, which detected a $73 \mathrm{kDa}$ band according to the manual. Protein levels were calculated by densitometry using actin as a normalization control.

A

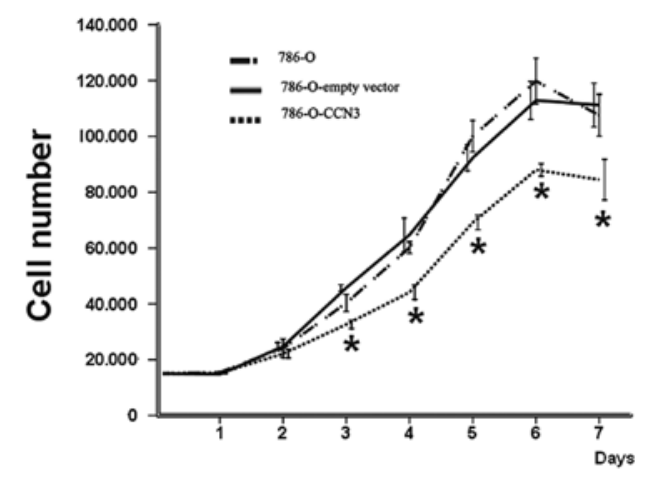

B

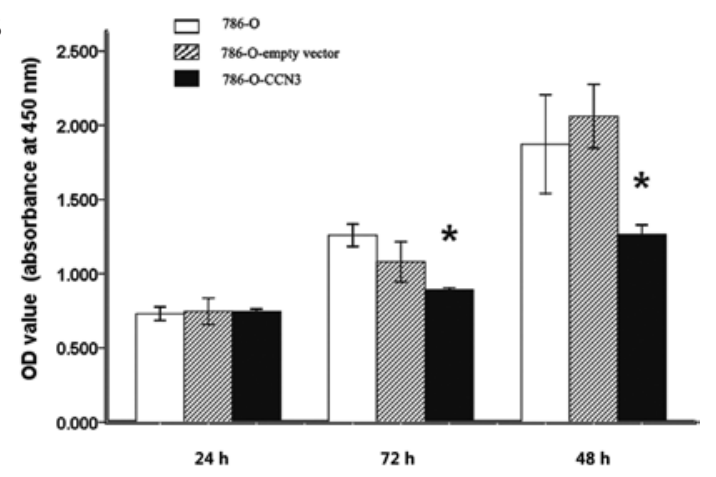

Figure 3. Effect of $\mathrm{CCN} 3$ expression on the proliferation of RCC cells in vitro. High expression of CCN3 decreases RCC cell proliferation. (A) Growth curve in vitro. (B) WST-1 assay at 24,48 and $72 \mathrm{~h}$. Data are shown as the mean \pm SD for three independent experiments. ${ }^{*} \mathrm{P}<0.05$ vs. $786-\mathrm{O}$ parental cells.
A

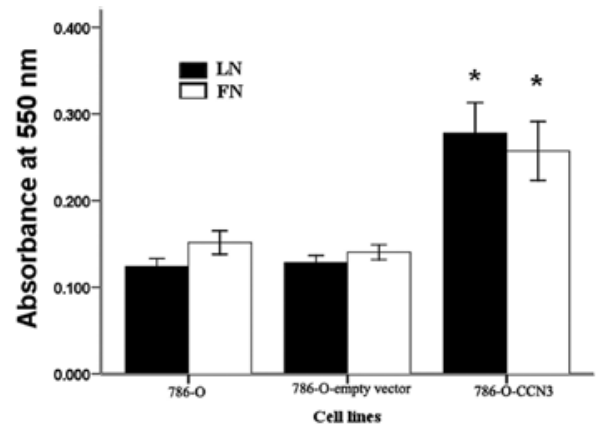

B

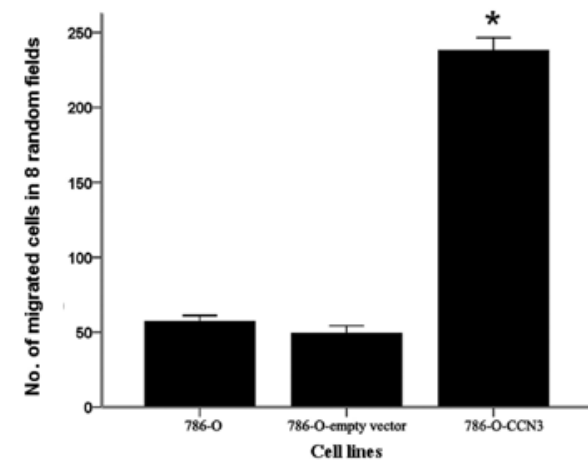

C

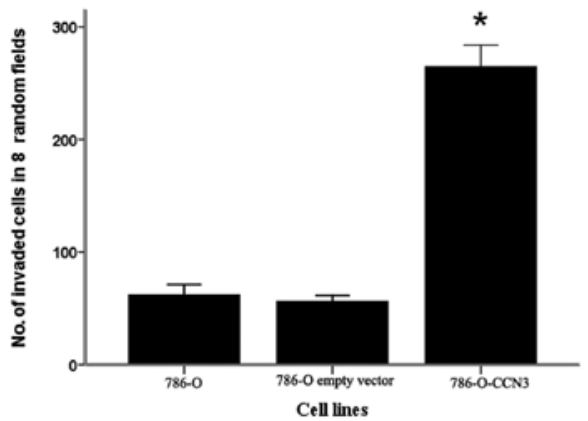

Figure 4. CCN3 promotes the adhesion, invasion and migration of RCC cells (A) Adhesion of RCC cells to ECM proteins. Cells were plated in wells coated with fibronectin (FN) and laminin (LN) according to the manual. After 90 min, adherent cells were stained with $0.1 \%$ crystal violet then washed by immersion in tap water and dried. Adhesion was measured as absorbance at $550 \mathrm{~nm}$. (B) For the migration assay, cells were seeded in the upper well of a transwell chamber. Medium with $10 \%$ fetal bovine serum was added to the lower well as the source of chemoattractants. Cells migrated through the polycarbonate membrane and were counted following $\mathrm{H} \& \mathrm{E}$ staining. (C) Invasion assays were performed using Matrigel-coated transwell chambers. The cells were embedded in Matrigel in the upper well, and the number of cells invading Matrigel and migrating to the lower well were counted following $\mathrm{H} \& \mathrm{E}$ staining. The data are the mean $\pm \mathrm{SD}$ of three independent experiments. ${ }^{~} \mathrm{P}<0.05$ vs. $786-\mathrm{O}$ parental cells.

found to be significantly increased in CCN3-transfected 786-O cells compared to the parental cells (Fig. 2).

In vitro growth features of CCN3-transfected clones. Our data show that a high expression of CCN3 significantly decreased the growth rate of $\mathrm{CCN} 3$-transfected 786-O cells, with a $22.07 \pm 1.97$ to $28.6 \pm 3.34 \%$ reduction at different time points compared to their parental cells, evident after three days of culture $(\mathrm{P}<0.05)$ (Fig. 3A).

The WST-1 proliferation assay was employed to test the proliferation of CCN3-transfected cells. The proliferation of 
CCN3-transfected cells was significantly decreased at $48 \mathrm{~h}$ $(\mathrm{P}<0.05$; percent inhibition, 17.45 $\pm 1.23 \%)$ and $72 \mathrm{~h}(\mathrm{P}<0.05$; percent inhibition, $36.12 \pm 6.92 \%$ ), compared to the parental cells (Fig. 3B).

Effects of CCN3 expression on the adhesive, migratory and invasive capabilities of 786-O cells. The effect of CCN3 expression on the adhesive properties of 786-O cells was analyzed with respect to the different ECM substrates, such as laminin and fibronectin. The results indicate increased adhesion of CCN3-transfected cells to fibronectin and laminin compared to the parental cells (Fig. 4A).

The migration of 786-O cells was measured in transwell chambers. CCN3-transfected cells exhibited a significant increase in cell migration ability compared to the parental cells $(\mathrm{P}<0.01)$ (Fig. 4B).

We further tested the ability of 786-O cells to invade through a reconstituted membrane barrier (Matrigel). Similarly to the migration assay, CCN3-transfected cells exhibited a significant increase in the invasive ability of these cells compared to the parental cells $(\mathrm{P}<0.01$, Fig. $4 \mathrm{C})$.

\section{Discussion}

The expression of the CCN3 gene has been found to be regulated temporally and spatially during chicken kidney development since high amounts of CCN3 were detected in embryonic kidneys, whereas much lower amounts were detected in the kidneys of birds after hatching, suggesting that $\mathrm{CCN} 3$ is related to the development of the kidney (15). In human kidneys, CCN3 expression is implicated in the differentiation of glomerular podocytes during normal nephrogenesis (4). High levels of CCN3 expression have been associated with heterotypic tumor differentiation in human nephroblastomas. Additionally, it has been suggested that overexpression of the $\mathrm{CCN} 3$ protein may be associated with tumor cell growth arrest (4). In the present study, we observed that the expression of CCN3 was lower in all clear cell RCC tissues compared to their corresponding normal kidney tissues using immunohistochemical staining. This finding is in line with previous findings obtained with quantitative RT-PCR in a panel of sporadic RCC tissues (16). In addition, we observed that the expression of $\mathrm{CCN} 3$ was inversely correlated with the Ki67 index in clear cell RCC tissues. Therefore, CCN3 may play an inhibitory role in clear cell RCC.

Since CCN3 was observed to be downregulated in clear cell RCC tissues, we transfected a clear cell RCC cell line (786-O) and obtained stable clones expressing the CCN3 gene to further study the role of CCN3 in the biology of clear cell RCC. We showed that the overexpression of CCN3 significantly inhibited the proliferation of clear cell RCC cells in vitro. This finding further indicated that $\mathrm{CCN} 3$ may be involved in the negative control of clear cell RCC cell growth.

However, $\mathrm{CCN} 3$ overexpression was found to promote clear cell RCC cell adhesion to ECM proteins, and clear cell RCC cell migration and invasion were increased through Matrigel. Our results are in line with previous findings obtained in Ewing's sarcoma (12). The expression of CCN3 by stable transfection significantly reduced the proliferative activity of Ewing's sarcoma cells, while increasing cell adhesion to ECM proteins and increased migration and invasion through Matrigel. Moreover, in a study by Manara et al (6), the expression of CCN3 was only observed in approximately $30 \%$ of cases of primary Ewing's sarcoma, but it was associated with a significantly higher risk of developing lung and bone metastasis. These findings supported the hypothesis that while an increase in proliferation is important for the initiation and maintenance of primary tumors, growth inhibition may be crucial for the survival of cancer cells in the circulation and secondary organs, thereby leading to the development of a more malignant phenotype (17). It is therefore conceivable that $\mathrm{CCN} 3$ plays a role in the metastasis of RCC.

Purified CCN3 has been shown to support adhesion and migration in endothelial cells and fibroblasts via integrins and heparan sulfate proteoglycans $(18,19)$. Despite the absence of an RGD sequence motif, CCN3 is a direct ligand of integrins $\alpha v \beta 3$ and $\alpha 5 \beta 1$ as demonstrated by solid phase binding assays (18). In Ewing's sarcoma, CCN3-transfected tumor cells exhibited a decreased expression of $\alpha 2 \beta 1$ integrin receptor, while cells lacking $\alpha 2 \beta 1$ are less less stably anchored to the ECM in Ewing's sarcoma tissue, resulting in increased migratory abilities (12). In melanoma, CCN3-transfected cells exhibited an increased expression of laminin and vitronectin integrin receptors $\alpha 7 \beta 1$ and $\alpha v \beta 5$, resulting in increased cell adhesion (20). Integrin subunits $\alpha 3, \beta 1, \alpha v, \alpha 2$ and $\alpha 5$ were highly expressed by $786-\mathrm{O}$ cells. The failure of $786-\mathrm{O}$ cells to organize $\beta 1$ fibrillar adhesions may be associated with their highly migratory phenotype (21). It is therefore conceivable that integrins may also be involved in CCN3-enhanced 786-O cell adhesion and migration. Further investigation is required to confirm the correlation between $\mathrm{CCN} 3$ and integrins in clear cell RCC.

In conclusion, our data indicate that $\mathrm{CCN} 3$ may play a significant role in clear cell RCC biology. The molecule exhibits anti-proliferative effects on tumor cells, while it promotes the adhesion, migration and invasion of clear cell RCC cells.

\section{Acknowledgements}

This study was supported by the Shandong Provincial Natural Science Foundation, China. We are grateful for the help and support of Dr Cecile Martinerie.

\section{References}

1. Rini BI and Atkins MB: Resistance to targeted therapy in renalcell carcinoma. Lancet Oncol 10: 992-1000, 2009.

2. Perbal B: NOV (nephroblastoma overexpressed) and the CCN family of genes: structural and functional issues. Mol Pathol 54: $57-79,2001$

3. Joliot V, Martinerie C, Dambrine G, et al: Proviral rearrangements and overexpression of a new cellular gene (nov) in myeloblastosis-associated virus type 1-induced nephroblastomas. Mol Cell Biol 12: 10-21, 1992.

4. Chevalier G, Yeger H, Martinerie C, et al: novH: differential expression in developing kidney and Wilms' tumors. Am J Pathol 152: 1563-1575, 1998.

5. Perbal B: The CCN family of cell growth regulators: a new family of normal and pathologic cell growth and differentiation regulators: lessons from the first international workshop on $\mathrm{CCN}$ gene family. Bull Cancer 88: 645-649, 2001.

6. Manara MC, Perbal B, Benini S, et al: The expression of ccn3(nov) gene in musculoskeletal tumors. Am J Pathol 160: 849-859, 2002.

7. Perbal B: The CCN3 protein and cancer. Adv Exp Med Biol 587: 23-40, 2006. 
8. Yu C, Le AT, Yeger H, Perbal B and Alman BAY: NOV (CCN3) regulation in the growth plate and $\mathrm{CCN}$ family member expression in cartilage neoplasia. J Pathol 201: 609-615, 2003.

9. Doghman M, Arhatte M, Thibout H, et al: Nephroblastoma overexpressed/cysteine-rich protein 61/connective tissue growth factor/nephroblastoma overexpressed gene-3 (NOV/CCN3), a selective adrenocortical cell proapoptotic factor, is downregulated in childhood adrenocortical tumors. J Clin Endocrinol Metab 92: 3253-3260, 2007.

10. Gupta N, Wang H, McLeod TL, et al: Inhibition of glioma cell growth and tumorigenic potential by CCN3 (NOV). Mol Pathol 54: 293-299, 2001.

11. Gellhaus A, Dong X, Propson S, et al: Connexin43 interacts with NOV: a possible mechanism for negative regulation of cell growth in choriocarcinoma cells. J Biol Chem 279: 36931-36942, 2004.

12. Benini S, Perbal B, Zambelli D, et al: In Ewing's sarcoma CCN3 (NOV) inhibits proliferation while promoting migration and invasion of the same cell type. Oncogene 24: 4349-4361, 2005.

13. Liu C, Liu XJ, Crowe PD, et al: Nephroblastoma overexpressed gene (NOV) codes for a growth factor that induces protein tyrosine phosphorylation. Gene 238: 471-478, 1999.

14. Maillard M, Cadot B, Ball RY, et al: Differential expression of the ccn3 (nov) proto-oncogene in human prostate cell lines and tissues. Mol Pathol 4: 275-280, 2001.
15. Perbal B: Contribution of MAV-1-induced nephroblastoma to the study of genes involved in human Wilms' tumor development. Crit Rev Oncog 5: 589-613, 1994.

16. Niu Z, Ito M, Awakura Y, et al: The expression of NOV and WT1 in renal cell carcinoma: a quantitative reverse transcriptase-polymerase chain reaction analysis. J Urol 174: 1460-1462, 2005.

17. Evdokimova V, Tognon C, Ng T and Sorensen PH: Reduced proliferation and enhanced migration: two sides of the same coin? Molecular mechanisms of metastatic progression by YB-1. Cell Cycle 8: 2901-2906, 2009.

18. Lin CG, Leu SJ, Chen N, et al: CCN3 (NOV) is a novel angiogenic regulator of the $\mathrm{CCN}$ protein family. J Biol Chem 278: 24200-24208, 2003.

19. Lin CG, Chen CC, Leu SJ, Grzeszkiewicz TM and Lau LF: Integrin-dependent functions of the angiogenic inducer NOV (CCN3): implication in wound healing. J Biol Chem 280: 8229-8237, 2005.

20. Fukunaga-Kalabis M, Martinez G, Telson SM, et al: Downregulation of $\mathrm{CCN} 3$ expression as a potential mechanism for melanoma progression. Oncogene 27: 2552-2560, 2008.

21. Esteban-Barragán MA, Avila P, Alvarez-Tejado M, et al: Role of the von Hippel-Lindau tumor suppressor gene in the formation of beta1-integrin fibrillar adhesions. Cancer Res 62: 2929-2936, 2002. 